\title{
Towards Adaptive Autonomous Robots in Autism Therapy: Varieties of Interactions
}

\author{
Kerstin Dautenhahn, Iain Werry, Tamie Salter, René te Boekhorst \\ Adaptive Systems Research Group \\ Department of Computer Science \\ University of Hertfordshire \\ United Kingdom \\ http://homepages .feis.herts.ac.uk/“nehaniv/ASRG.html
}

\begin{abstract}
This paper reports results deriving from the Aurora project (www.aurora-project.com) where we have pioneered research into the possible use of robots in autism therapy. Autistic children have difficulties in social interaction, communication and fantasy and imagination. As part of the project we run trials where autistic children are playing with a small, non-humanoid mobile robot that can engage children in simple interaction games. In our project we focus on the behavioural, rather than the affective level of robots used in therapy, cf. [12]. In this paper we first discuss in more detail varieties of interactions where one child, or two children simultaneously, play with a robot. We then outline a new research direction in the project which studies how a mobile robot can adapt to individual children. Quantitative examples of activity levels in child-robot interactions are included. The paper concludes by outlining future research directions for adaptive robots in autism therapy.
\end{abstract}

\section{Introduction: Autism}

Autism is a developmental disorder that effects about 5-15 in 10000 people. All individuals diagnosed with autism will show impairments in social interaction, communication, and imagination and fantasy. In addition, we find restricted repetitive and stereotyped patterns of behaviour, interests, and activities. For people autism it is often difficult to cope with novel situations or interruptions of their daily routine. Moreover, they tend not to show proactive behaviour in social situations, e.g. they need to be taught explicitly to ask when they require help. However, individuals with autism present a broad spectrum of difficulties and abilities, and vary enormously in their levels of overall intellectual functioning. Many therapy methods exist for autism, see review in [5]. But some therapy methods seem to work better for a particular child with autism than others.

To an autistic person other people's social behaviour often appears overwhelming, unpredictable, confusing and therefore threatening. Interestingly, it has been shown that computers can provide a predictable, 'safe', and thus enjoyable environment ([2], [16], [11]). Also, people with autism usually focus on the 'literal meaning' and details of things, rather than perceiving the world in a holistic and socially and culturally interpreted sense. For example, in our very first trials where children with autism interacted with a robot, we discovered the vital importance to remove all small wires or other features that seemed to us 'unnoticible', but which autistic children focussed on (e.g. trying to inspect or pull out the wires). The simplified world of computers and other machines is much closer to a perspective of 'literal meaning'. Because computers follow simple rules in a predictable way their behaviour requires less 'interpretation' than e.g. a human's facial or verbal expressions, or gestures.

\section{Robot Technology in Autism Therapy}

\subsection{Background}

Using robots in education has been an active area of research for over thirty years [13], [8]. With an increasing interest in service robots, the area of using robots in therapeutic contexts has emerged. An example is the robotic seal robot Paro that has been developed for robot assisted activity for elderly people [19].

Increasingly researchers study the application of interactive software and robotic systems to autism therapy, cf. early work with a teleoperated (non-interactive) robotic turtle [20]. More recently interactive systems, namely virtual environments ([18], [14]), computer technology $([1])$, and robotic systems are being studied [3], $[10],[15]$. For more discussions on the role of different robotic designs in autism therapy see [4].

\subsection{Why robots?}

Why could robots be interesting tools or toys in autism therapy? Isn't it so much easier to create interactive software agents for this purpose? In our view robots have the following advantages:

1. Robots allow simplified, but embodied interaction, involving touch, physical manipulation (e.g., carrying the robot) etc. Thus, interaction with a robot is more 'real' than interacting with an agent on a computer screen, but less 'real' than interacting with another human being. Thus, robots might bridge the gap between software systems that are being used in autism therapy (e.g. in order to exercise a variety of different skills), and interactions with teachers, parents, and others.

2. In terms of abstraction, robots are again in between the software world and the real world: Interacting with them is less abstract than interacting with a virtual agent, but more abstract than interacting with another human being. 
3. Due to the situatedness and embodiment of robotchild interactions, interaction dynamics play a vital role. Whatever programmes are controlling the robot, interactions with the children are real-time. Neither the robot, nor the child can escape from the interactions dynamics, e.g. the world cannot be halted or frozen, or played backwards etc.

4. Robots 'naturally' support multi-modal interaction, including touch.

\subsection{Aurora: The importance of play}

Our general approach towards robot-child interactions in the Aurora projet is inherently playful, cf. figure 1. The enjoyment of the children, i.e. the pleasure they usually visibly experience when playing with the robot (smiles, laughs etc.), is used as the 'hook' that lets them being engaged with the robot for a particular period of time. No explict goals, tasks, or rewards are provided.

What robotic behaviours are therapeutically relevant and can be addressed during play with a mobile robot? In the Aurora project we target fundamental concepts of communication and interaction dynamics, such as:

- proactive behaviour

- communication with other children and adults (cf. [7])

- turn-taking

- coping with novelty

- play skills (more specifically addressed in [22])

- eye gaze and attention in communication and interaction (cf. [6], [21])

- imitation (cf. section below, as well as initial trials with a humanoid robotic doll that can imitate arm movement of children [4])

In the autism literature, people with autism are said to have certain impairments in all of the above mentioned concepts.

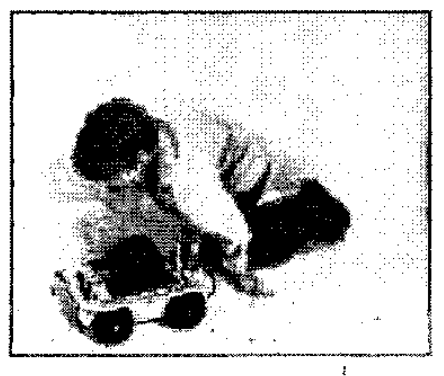

Figure 1: An autistic boy playing with a mobile robot (Labo-1, donated by Applied AI Systems. The size of the robot is $38 \mathrm{~cm}$ by $30 \mathrm{~cm}$ by $21 \mathrm{~cm}$. It weighs 6.5 kg.).

\subsection{Previous results}

When we started the Aurora project in 1998 no related work on interactions of autistic children with a mobile, autonomous robot were available. We therefore had to conduct a number of 'ground work' studies, including the development of appropriate evaluation techniques. Below we summarise the main findings that we published previously.

In various studies involving dozens of children with autism we established:

- The mobile robot that we used provided an enjoyable and safe play environment. The children were not afraid of the robot and could well cope with the novel situation when being exposed to the robot for the first time.

- The children were more attracted towards the mobile robot in comparison to a non-robotic toy. This comparative study revealed that the children showed significantly more eye-gaze and attention directed towards the robot. They also seemed to exhibit more pro-active behaviour when being exposed to the robot. In this study we had to develop a quantitative technique for evaluating robot-human interactions, see [6].

- In pair trials where two children interacted simultaneously with the robot different play styles could be observed. Depending on their social skills we observed behaviour ranging from non-social or competitive play to social play. This dual child scenario points towards using a robot as a social mediator that might encourage children with autism to play with each other, see [22], and below.

- We observed instances where children with autism used the robot in order to make contact to adults (teacher or experimenter). This involved very subtle behaviours that could only be detected with an in-depth quantitative evaluation technique, see [7].

The robot that we used in the studies summarised above was a Labo-1 robot (Applied AI Systems), a very robust mobile robot with eight infrared sensors and one heat sensor, cf. figure 1 .

Using interactive systems in autism therapy is a very recent development, and therefore research in that area often has an exploratory character. As we argued previously, important progress in the area will depend on systematic qualitative and quantitative evaluations that can reveal any therapeutic benefits. In the area of autism therapy the use of questionnaires, which are being used widely in robotics in order to assess subjective experience of subjects who interact with robots (e.g. [17]) is not appropriate. See [7], [6] for more discussion of evaluation methods for using robots in autism therapy.

However, given the results from our previous studies, what is a useful next step forward? In this paper we argue that interesting research directions can be revealed by analysing the spectrum of varieties of interactions that can occur. The next section gives a few selected examples.

\section{Varieties of Interactions with a Mobile Robot}

In this section we describe examples of interactions that occurred when autistic children interacted with a mobile 
robot. All subjects were boys, the trials were conducted at the children's school. The room that we used was about $3 \mathrm{~m}$ by $4 \mathrm{~m}$ in size, it was empty apart from chairs (for the experimenter).

We distinguish single and dual child trials. In the former case a single child is exposed to a mobile robot. The set up of the trials and a description of the robot that was used is given in more detail in [6] (for single child trials) and in [22] (dual child trials). Children and robot are not alone in the room, a teacher is present who the children are familiar with, as well as an experimenter. The adults do not initiate interactions with the children, but they do respond when being addressed by the children. Thus, the trial set up is inherently social, and indeed we showed how this might reveal communicative and interactive competencies of children with autism ([7]). A variety of play, interaction and communication styles can be observed, and examples are given below. In both cases the robot performs simple chasing and following games with the children, using its infrared and pyrosensors. Such games are therapeutically useful since they incorporate the concept of turn-taking which is central to human communication, interaction and dialogue in general, and which is therefore also taught explicitly in schools for children with autism. The transcripts below indicate the time stamps (minutes and seconds after start of the trial).

\subsection{Single child trials}

\section{Transcript 1:}

00m02s Child begins session with the robot directly in front of him, while he is standing. The child then backs away three steps from the robot and stands stationary.

$00 \mathrm{m08s}$ The robot orientates itself and then moves towards the child.

00m10s The child moves a small distance backwards, against the room wall, continuously watching the robotic platform.

00m15s When the robot does not move closer to the child, he moves towards the robot a short distance, until the robot reacts and backs away from the child. The robot then rotates but remains the same distance from the child.

00m23s Again, the child moves a little towards the robot until it reacts and retreats.

$00 \mathrm{~m} 26 \mathrm{~s}$ The child changes direction and again backs away from the robot to observe its reaction. When the robot follows the child, he continues his movement away from the robot. The robot remains a set distance from the child, who is now against the wall again

00m37s The child turns sideways to present his right side to the robot.

$00 \mathrm{m45s}$ The robot turns too far and faces the area now behind the child. The child takes a step backwards to place himself in front of the robot.

00m48s The child takes a second step backwards, in order to allow the robot to follow him, which it does.

00m53s The child stamps his feet and moves towards the robot, which moves away quickly.

$00 \mathrm{m58s}$ The child sits down in front of the robot and observes its movement.

$01 \mathrm{m02s}$ The child reaches out to the robot but is not close enough to touch it.

01 m08s The robot moves towards the child.

$01 \mathrm{~m} 10$ s The child again reaches out to the robot, which backs away from the child's outstretched hand.

$01 \mathrm{m17s}$ The child looks at the experimenter and smiles as he touches the robot.
Note, that the robot's behaviours are simple, but not completely predictable. This issue is important since using robots in autism therapy should not perpetuate existing repetitive tendencies. The example above clearly shows the variety of behaviours that the robot elicits and mediates:

- The child pays acute attention to the robot's behaviour, even in cases when he is just watching its behaviour from a distance.

- The child is not afraid of the robot, i.e. he does not show any signs of distress during the interactions with the robot, even when he is standing against the wall $[00 \mathrm{~m} 10 \mathrm{~s}],[00 \mathrm{~m} 26 \mathrm{~s}]$.

- The child reacts promptly when the robot moves towards him.

- The child quickly, namely within the first $15 \mathrm{sec}-$ onds, finds out how to operate the robot, e.g. how to make it back away.

- Rather then purely reacting to any actions of the robot, the child proactively explores how to interact with the robot, seemingly as if he is testing an hypothesis regarding the robot's behaviour and then waits for the robot to confirm or disconfirm (e.g. $[00 \mathrm{~m} 10 \mathrm{~s}],[00 \mathrm{~m} 15 \mathrm{~s}],[00 \mathrm{~m}, 45 \mathrm{~s}],[01 \mathrm{~m} 10 \mathrm{~s}])$.

- The child experiments with the robot by confronting the robot with different stimuli, e.g. presenting his side to the robot $[00 \mathrm{~m} 37 \mathrm{~s}]$, or stamping his feet [00m53s]. Again, this behaviour can be interpreted in the context of a child 'experimenter'.

- The child is proactively trying to keep the interaction going, 'helping' the robot by moving closer etc., for example [00m $23 \mathrm{~s}],[00 \mathrm{~m} 45 \mathrm{~s}]$.

- Although the robot's behaviour repertoire is very limited, the child does not loose interest.

- The child is visibly enjoying the interactions (smiling) $[01 \mathrm{~m} 17 \mathrm{~s}]$

- The child is sharing his enjoyment with an adult (an unfamiliar experimenter) [01m17s].

The next transcript gives an example of how a child guides the robot using his knowledge of the robot's obstacle avoidance behaviour.

\section{Transcript 2:}

02m58s Child is kneeling in front of the robot, which is facing him, and pressing the buttons at the rear of the platform. As he leans forward, the robot backs away.

$03 m 01 s$ The child follows the robot forwards and then restrains the robot by holding onto the sides to stop its movement.

03 m02s The child lets go of the robot, which moves away from him. The child then moves a chair out of the robots path as it reverses away from the child.

03m05s The child forcefully moves the robot away from the wall as it becomes stuck between the wall and the chair.

03m07s The child again kneels in front of the robot and places his arms either side of the platform, forcing the robot to retreat directly away from the child.

$03 \mathrm{m10s}$ As the robot moves away, the child follows, always obstructing the robot on three sides. 
03m13s The robot moves out of the child's reach, and towards the door.

03m16s The child approaches the robot, directing it out of the room through the door.

03m18s The child holds the door open to allow the robot to pass through.

03m27s The child picks up the robot and brings it back into the centre of the room.

Note, that the child in the above example uses his whole body in order to play with the robot, utilising his knowledge of how to operate the robot. Moreover, the child can predict the robot's behaviour, 'helping' the robot by moving obstacles out of the way [03m02s].

\subsection{Dual child trials}

The transcript below describes a scenario where two children were simultaneously exposed to the robot. The children in these trials were paired by the teachers according to their social skills. Spencer and Adam are classmates, quite social and verbal autistic children.

Spencer and Adam were part of a larger study involving three pairs of children. As reported in [22], the pair trials showed interesting aspects of robot-child and child-child interactions:

- The social abilities that the children exhibited during the trials with the robot matched the social abilities they show in the classroom or other contexts.

- Depending on the children's social abilities, different play styles could be observed, ranging from social play (Adam and Spencer) to non-social play (the children are playing nicely with the robot but not with each other, occasional child-child interaction is due to the fact that they compete for the robot's 'attention'), and non-social play (children try to keep each other away from the robot).

\section{Transcript 3:}

$07 \mathbf{m 4 8 s}$ Spencer stands in front of the robot in an effort to instruct it to retreat. The robot does not move and does not appear to sense the child.

$07 \mathrm{m50}$ sxperimenter approaches the robot and informs the child that "you can chase it". She places her hand in front of the robot and it backs away. Spencer observes this behaviour, and exclaims "oh yeah".

$07 \mathrm{m57}$ spencer then crouches in front of the robot and attempts to imitate. However, he places his hand in front of the heat sensor and not the infrared sensors. As a result the robot moves forwards, towards the heat, and cannot sense an object to avoid with the infrared sensors.

$08 \mathrm{m03s}$ When it becomes obvious that the action is not having the desired effect, Spencer states "I cant do it".

08 m04s The second experimenter then offers the opinion that "maybe its not frightened of you".

$08 m 09$ s The first experimenter then suggests that the child should 'be lower' and again demonstrates by directing the object backwards.

$08 \mathrm{m12s}$ The child then approaches the robot and crouches down again. He waves his hand in front of the robot and the platform moves backwards. The experimenter encourages this action.

Then, later in the session, Spencer demonstrates this skil Adam.

$09 \mathrm{m07}$ s Spencer operates the robot by waving his hand in front of the infrared sensor. Adam then asks 'how do you make it do that?'.
$09 \mathrm{~m} 11$ s Spencer replies 'if you want it to go away, you've got to put your hand lower down and when it sees your hand then it goes away', and continues to demonstrate.

This example shows a variety of interesting childchild and child-adult (experimenter) interactions:

- Adult-child 'teaching': An adult demonstrates how to operate the robot $[07 \mathrm{~m} 50 \mathrm{~s}]$, the child tries it out himself and then notices that his attempt was unsuccessful $[07 \mathrm{~m} 57 \mathrm{~s}]$. After another demonstration by the experimenter [08m09s] the child succeeds $[08 \mathrm{~m} 12 \mathrm{~s}]$. Note, that this required the child to monitor and correct his own actions.

- Child-child 'teaching': After having learnt a new behaviour from the experimenter the child later demonstrates this skill to another child $[09 \mathrm{~m} 07 \mathrm{~s}]$ and explicitly gives instructions [09m11s].

- Adults in the room (in this case an unfamiliar experimenter) are used as sources of information.

In addition to child-robot interactions that could be therapeutically useful and enjoyable, this example also shows an important role of the robot as a mediator that mediates child-child and child-adult interactions. We explain the concept of a robot mediator in more detail in [22]. This is particularly useful since it can help with the generalisation of skills learnt in robot-human interactions to human-human interactions. Note, that generalisation is a particularly severe problem in autism therapy in general (children tend to strongly limit newly learnt skills to the particular context in which they were learnt).

\section{Adaptive Robots in Autism Therapy}

Studies by Ferrara and Hill [9] compared how children with autism interact with different types of (nonrobotic) toys. Results showed that in contrast to control groups with typically developing children, autistic children prefer simple designs in relatively predictable environments. Ferrara and Hill conclude that those form an excellent starting point for therapeutic intervention where one could slowly increase the complexity of the therapeutic toys.

A more appropriate starting place for therapeutic intervention with autistic children might be to focus on their development of social play. Social objects with low intensity should first be presented in a game that has a highly predictive and repetitive sequence of activities. Complexity of social stimuli and game activities should gradually increase in intensity. When the child begins to show pleasure in these games and to initiate them, the introduction of language and cognitive tasks matching the complexity of the game would be appropriate. [9], p. 56 .

Thus, toys in autism therapy need to grow and develop alongside the children, with respect to the children's social, emotional and therapeutic needs. Most importantly, such toys need to be able to recognize individual differences among the children (e.g. play or other interaction styles), so that they can adapt their own behaviour accordingly. One important characteristic of robot-human interaction is touch, which has been used 
previously as a valuable quantitative measure for robothuman interactions, e.g. in our work [6], or in other work in robot assisted therapy [19]. In the following we describe touch interaction in order to characterise the activity levels of children in robot-child interaction.

\subsection{Experiments: Assessing children's ac- tivity levels}

In order to meet Ferrara and Hill's criteria of therapeutic toys that can change, in a new research direction within the Aurora project we develop robots that can adapt to how individual children interact with robots. We use a robotic mobile robot platform called Pekee (see figure 2) that possesses fifteen infrared sensors (sensor positions shown in figure 3), two gyrometers, two temperature sensors, one shock sensor, and one light sensor. In our robotics laboratory we conducted an initial study with three 5-6 year old typically developing children (boys: Colin, Tom, Victor). We hypothesized that activity levels are influenced by the children's personalities, and therefore asked the parents to classify their child as either shy, boisterous, or neutral. Only one child (Colin) was classified as shy.

We let the children interact with Pekee for two minutes each. In this setup, Pekee is running in a $2 \mathrm{~m}$ by 2 $\mathrm{m}$ arena, surrounded by $40 \mathrm{~cm}$ high plywood walls. The arena is empty, apart from the robot and children who might enter the arena. The infrared sensors reach up to $3 \mathrm{~m}$ and are used for obstacle avoidance. This basic behaviour works reliably so that, in the absence of any children in the arena, the sensors never detect any object at very close distance (i.e. below approximately 25 $\mathrm{cm}$ ). We defined a $2 \mathrm{~cm}$ zone around the robot where any detected object is classified and remembered as a 'touch'. The interactions of children with the robot are videotaped for reference purposes. In an initial test we confirmed that any detection event of less than $2 \mathrm{~cm}$ corresponds to a person touching Pekee.

In our experiments, each child is brought separately into the arena and given the instruction "go in an play and do whatever you want". The robot runs a basic obstacle avoidance programme. The infrared sensors are sampled at a frequency of about $20 / \mathrm{sec}$. The sensor readings of all $15 \mathrm{IR}$ sensors are stored and later analyzed. Figure 4 shows the activity levels of three children. While two of the children interact very actively with the robot, Colin's data reflects very low interaction/touch intensity (the maximum for Colin is four instances of touch, compared to 590 for Victor and 289 for Tom). In total Colin touched the robot 24 times during the two-minute period, in comparison to 725 (Tom) and 4330 (Victor).

\subsection{Discussion}

Although the data presented in the previous section is preliminary, it shows different activity levels of children interacting with the robot. The shy/boisterous classification of the children is reflected in the videodata where we see that shy children behaved less confidently towards the robot than boisterous children. But interestingly, the shy/boisterous distinction is also apparent in the robot's sensor data. Colin, who had been classified as being shy, showed very low activity levels in comparison to the other two children. Note, that in figure 4 Colin's activity levels are so low that they are hardly visible.

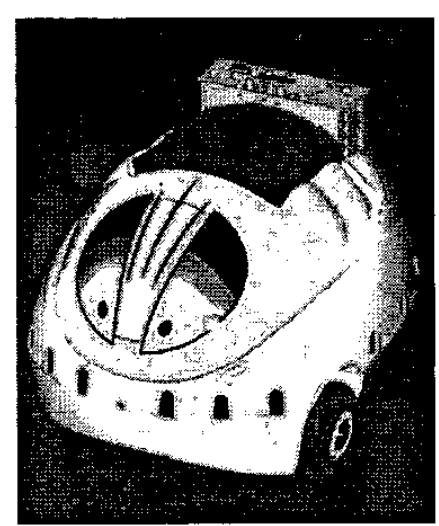

Figure 2: The mobile robot Pekee (Wany Robotics) used in our experiments on activity levels.

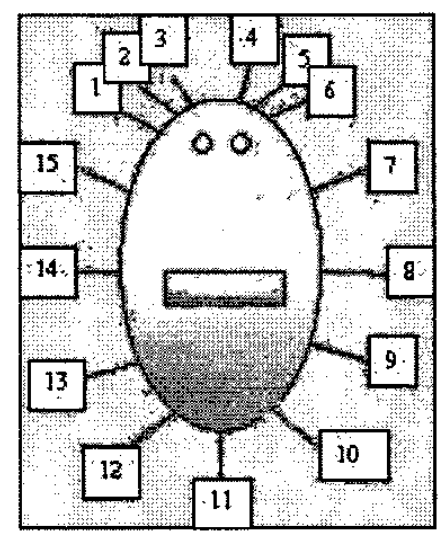

Figure 3: Position of Pekee's 15 infrared sensors.

\section{Comparison of activity levels}

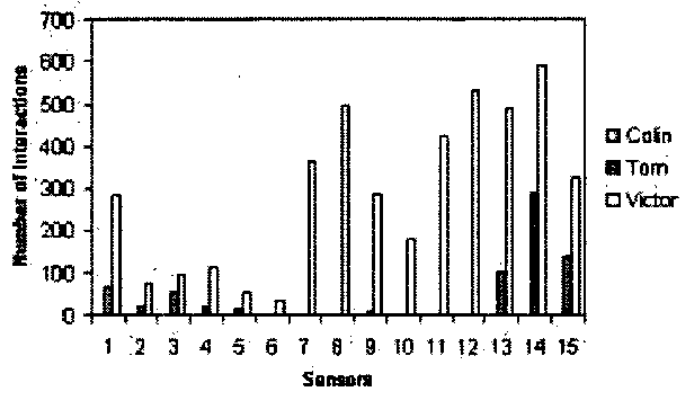

Figure 4: Activity levels of three children interacting with Pekee. Colin is a 5-year old boy, Tom and Victor are age six (all names are pseudonyms). The horizontal axis represents Pekee's 15 infrared sensors. The vertical axes shows the total number of touch events for each sensor during the 2-minute test interval. 
In ongoing trials we use a larger number of nonautistic and autistic children, with repeated exposures to the robot in order to test the hypothesis that a) by means of the robot's sensor readings distinct activity levels can be recognised for each child, and b) that these levels are consistent over repeated exposures (the experiments we are currently running seem to give evidence for this). Also, we are investigating a variety of statistical methods for analyzing the sensor data. More specifically, based on the activity level data, we are interested in how distinct and individual interaction styles/patterns can be detected. These styles/patterns could then serve as a source for an adaptive robot.

\section{General Conclusions and Outlook}

This paper showed a variety of child-robot and childchild interactions that can be encouraged and mediated by a mobile robot. Future work will include long-term studies that investigate any therapeutic effects of using robotic toys for autistic children. Another important direction for future research will be to develop robots that adapt to individual characteristics of children, and that remember, and act upon interaction histories with children. We hope that such adaptive robots will be an important step towards the development of effective therapeutic robots in autism therapy, a goal that we have been pursuing for the past five years.

\section{References}

[1] Katherine H. Blocher. Affective Social Quest (ASQ) Teaching emotion recognition with interactive media and wireless expressive toys. Master's Thesis for Master of Science in Media Technology Massachusetts Institute of Technology, MIT, USA, 1999.

[2] K. M. Colby and D. C. Smith. Computers in the treatment of nonspeaking autistic children. Current Psychiatric Therapies, 11:1-17, 1971.

[3] Kerstin Dautenhahn. Robots as social actors: Aurora and the case of autism. In Proc. CT99, The Third International Cognitive Technology Conference, August, San Francisco, pages 359-374, 1999.

[4] Kerstin Dautenhahn and Aude Billard. Games children with autism can play with robota, a humanoid robotic doll. In S. Keates, P. M. Langdon, P.J. Clarkson, and P. Robinson, editors, Universal Access and Assistive Technology, pages 179-190. Springer-Verlag London, 2002.

[5] Kerstin Dautenhahn and Iain Werry. Issues of robotbuman interaction dynamics in the rehabilitation of children with autism. In J.-A. Meyer, A. Berthoz, D. Floreano, H. Roitblat, and S. W. Wilson, editors, Proc. From animals to animats 6 , The Sixth International Conference on the Simulation of Adaptive Behavior (SAB2000), pages 519-528, 2000.

[6] Kerstin Dautenhahn and Iain Werry. A quantitative technique for analysing robot-human interactions. In Proc. IEEE/RSJ International Conference on Intelligent Robots and Systems (IROS 2002), pages 11321138. IEEE Press, 2002.

[7] Kerstin Dautenhahn, Iain Werry, John Rae, Paul Dickerson, Penny Stribling, and Bernard Ogden. Robotic playmates - analysing interactive competencies of children with autism playing with a mobile robot. In K. Dautenhahn, A. Bond, L. Ca namero, and B. Edmonds, editors, Socially Intelligent Agents - Creating Relationships with Computers and Robots, pages 117124. Kluwer Academic Publishers, 2002.
[8] Allison Druin and James Hendler, editors. Robots for Kids - Exploring new technologies for learning. Morgan Kaufmann Publishers, 2000.

[9] Cindy Ferrara and Suzanne D. Hill. The responsiveness of autistic children to the predictability of social and non-social toys. Journal of Autism and Developmental Disonders, 10(1):51-57, 1980.

[10] F. Michaud, A. Clavet, G. Lachiver, and M. Lucas. Designing toy robots to help autistic children - an open design project for electrical and computer engineering education. Proc. American Society for Engineering Education, 2000.

[11] D. Moore. Computers and people with autism. Communication, pages 20-21, 1998.

[12] Tatsuya Nomura and Noriyuki Tejima. Critical considerations of applications of affective robots to mental therapy from psychological and sociological perspectives. In Proc. 11th IEEE Int. Workshop on Robot and Human Interactive Communication (ROMAN 2002), pages 99104. IEEE Press, 2002.

[13] Seymour Papert. Mindstorms: Children, Computers, and Powerful Ideas. Basic Books, New York, 1980.

[14] S. Parsons, L. Beardon, H. R. Neale, G. Reynard, R. Eastgate, J. R. Wilson, S. V. Cobb, S. D. Benford, P. Mitchell, and E. Hopkins. Development of social skills amongst adults with asperger's syndrome using virtual environments: the 'as interactive' project. In Paul Sharkey, Antonio Cesarani, Luigi Pugnetti, and Albert Rizzo, editors, Proc. The 3rd International Conference on Disability, Virtual Reality and Associated Technologies, ICDVRAT 2000, 23-25 September 2000, Alghero, Sardinia, Italy, pages 163-170, 2000.

[15] C. Plaisant, A. Druin, C. Lathan, K. Dakhane, K. Edwards, J. M. Vice, and J. Montemayor. A storytelling robot for pediatric rehabilitation. Proc. ASSETS '00, Washington, Nov. 2000, ACM, New York, pp. 50-55, 2000.

[16] S. Powell. The use of computers in teaching people with autism. In: Autism on the agenda: papers from a National Autistic Society Conference, London, 1996.

[17] Takanori Shibata, Kazuyoshi Wada, and Kazuo Tanie. Tabulation and analysis of questionnaire results of subjective evaluation of seal robot at science museum in london. In Proc. 11th IEEE Int. Workshop on Robot and Human Interactive Communication (ROMAN 2002), pages 23-28. IEEE Press, 2002.

[18] D. Strickland. A virtual reality application with autistic children. Presence: Teleopemators and Virtual Environments, 5(3):319-329, 1996.

[19] Kazuyoshi Wada, Takanori Shibata, Tomoko Saito, and Kazuo Tanie. Analysis of factors that bring mental effects to elderly people in robot assisted activity. In PToc. IEEE/RSJ International Conference on Intelligent Robots and Systems (IROS 2002), pages 11521157. IEEE Press, 2002.

[20] S. Weir and R. Emanuel. Using LOGO to catalyse communication in an autistic child. Technical report, DAI Research Report No. 15, University of Edinburgh, 1976.

[21] Iain Werry, Kerstin Dautenhahn, and William Harwin. Investigating a robot as a therapy partner for children with autism. Proc. AAATE 2001, 6th European Conference for the Advancement of Assistive Technology (AAATE 2001), 3-6 September 2001 in Ljubljana / Slovenia., 2001.

[22] Iain Werry, Kerstin Dautenhahn, Bernard Ogden, and William Harwin. Can social interaction skills be taught by a social agent? the role of a robotic mediator in autism therapy. In M. Beynon, C. L. Nehaniv, and K. Dautenhahn, editors, Proc. CT2001, The Fourth International Conference on Cognitive Technology: Instruments of Mind, LNAI 2117, pages 57-74, Berlin Heidelberg, 2001. Springer-Verlag. 